\title{
Prognostic Alternative mRNA Splicing in Adrenocortical Carcinoma
}

\section{OPEN ACCESS}

Edited by:

Ruth Andrew,

University of Edinburgh,

United Kingdom

Reviewed by:

Christopher Gerner,

University of Vienna, Austria

Tom Michoel,

University of Bergen, Norway

${ }^{*}$ Correspondence:

Weiwei Liang

helenliangww@zju.edu.cn

tThese authors have contributed equally to this work and share first authorship

Specialty section:

This article was submitted to Systems Endocrinology, a section of the journal Frontiers in Endocrinology

Received: 28 February 2020 Accepted: 10 February 2021 Published: 12 March 2021

Citation:

Liang W and Sun F (2021) Prognostic Alternative $m R N A$ Splicing in Adrenocortical Carcinoma.

Front. Endocrinol. 12:538364. doi: 10.3389/fendo.2021.538364

\begin{abstract}
Weiwei Liang ${ }^{1 \text { }}{ }^{\text {at }}$ and Fangfang Sun ${ }^{2 \dagger}$
${ }_{1}^{1}$ Department of Endocrinology, The Second Affiliated Hospital, Zhejiang University School of Medicine, Hangzhou, China, 2 Key Laboratory of Cancer Prevention and Intervention, China National Ministry of Education, The Second Affiliated Hospital, Cancer Institute, Zhejiang University School of Medicine, Hangzhou, China
\end{abstract}

Background: This paper aims to identify alternative RNA splicing landscape and its prognostic value in adrenocortical carcinoma.

Methods: The alternative splicing events data with corresponding clinical information data of 79 ACC patients were obtained from the Cancer Genome Atlas and SpliceSeq package. Prognosis-associated AS events by using univariate Cox regression analysis were selected. Gene functional enrichment analysis demonstrated the potential pathways enriched by survival-associated AS. Prognosis-related splicing events were submitted to develop moderate predictors using Lasso regression model.

Results: One thousand five survival-associated alternative splicing events were identified. The prognostic genes included ATXN2L, MEIS1, IKBKB, COX4I1. Functional enrichment analysis suggested that prognostic splicing events are associated with Wnt signaling pathway. A prediction model including 12 alternative splicing events was constructed by Lasso regression using train set. ROC analysis showed good performance of the prediction model in test set. Then, a nomogram integrating the clinical-pathological factors and riskscore was constructed for predicting 1- and 3-year survival rate.

Conclusion: Our data provide a comprehensive bioinformatics analysis of AS events in ACC, providing biomarkers for disease progression and a potentially rich source of novel therapeutic targets.

Keywords: alternative splicing, adrenocortical carcinoma, AUC, bioinformatics, prognostic model

\section{INTRODUCTION}

Cancers are often associated with aberrant proteins. Cancer genome research showed the number of protein-coding genes was limited, which makes it difficult to account for the diverse proteomic phenotypes. Alternative RNA splicing is a key step of post-transcriptional gene expression regulation (1). There were seven types of alternative splicing events: Exon Skip (ES), Mutually Exclusive Exons (ME), Retained Intron (RI), Alternate Promoter (AP), Alternate Terminator (AT), Alternate Donor site (AD), and Alternate Acceptor site (AA). Through alternative processing, genes can produce distinct RNA isoforms. Alternative splicing contributes to the protein diversity for $90 \%$ of human gene expression. Thus, it is not surprising that defects in alternative splicing are involved in tumorigenesis (2). AS also provided targets of cancer treatment (3). Characterization of the AS landscape provides a wealth of insight into cancers with excellent prognostic value. 
Our study aimed to detect AS landscape in adrenocortical carcinoma (ACC). ACC is a rare endocrine tumor with generally poor prognosis. Study showed median overall survival (OS) is 3.21 years, and 5-years overall survival of ACC is below $40 \%$ in most series (4-9). ACC counts $0.2 \%$ of all cancer deaths in the United States, while the incidence of ACC is only 0.72 per million cases per year (10). The prognosis of ACC is very heterogeneous. Identifying prognostic factors of ACC is significant for clinical decision making. The recent studies had revealed some prognosis-associated molecular mechanisms of ACC (11). However, studies of alternative splicing in ACC are still lacking.

To unravel AS landscape and its prognostic value in ACC, this study utilized the rich data from The Cancer Genome Atlas (TCGA) consortium. We systematically profiled the alternative splicing events in ACC cohort from TCGA. Survival-associated alternative splicing events were identified. Then, we constructed prognostic model for ACC patients.

\section{METHODS}

\section{Data Sources}

The RNA sequencing data with corresponding clinical information data of 79 ACC patients were obtained from the Cancer Genome Atlas (https://tcga-data.nci.nih.gov/tcga/) and SpliceSeq package $(12,13)$. Clinical information data included age, gender, TNM stage, overall survival (OS), and live/dead status. Totally seven types of alternative splicing events were all involved in our study.

\section{Survival Analysis}

The flow chart for the experimental design is displayed in Figure 1. Univariate Cox regression was first used to access the association between all alternative splicing events and OS. Multiple testing correction was done using Bonferroni adjustment. P. adj $<0.05$ was set as the cut-off criterion. Data set was divided into training set and texting set. A least absolute shrinkage and selection operator (LASSO) penalized Cox regression model was used to select the most useful alternative splicing events as prognostic predictors. A prognostic model was then constructed based on the percentspliced-in (PSI) level of the selected alternative splicing events using Cox regression coefficients. The patients were classified into low-risk group and high-risk group according to the median value of the risk scores. The area under the curve (AUC) of the receiveroperator characteristic (ROC) curve for prognostic model was generated. The survival outcome endpoint was selected as 1 year and 3 years because fewer events occurred after 3 years in survival analyses. All reported $\mathrm{p}$ values were two-sided. $\mathrm{p}<0.05$ was set as the cut-off criterion.

All analyses were performed using Bioconductor in R (version 3.5.3). Upset plot was done using the "UpsetR" package (14). Univariate Cox regression was done using the "survival" package (15). The LASSO analysis was undertaken using the "glmnet" package (16). ROC curve was performed with the function of “survivalROC” (17).

\section{Functional Analysis}

We used Gene ontology analysis (GO) to identify characteristic biological attributes of the genes with survival-associated AS events. We performed Kyoto Encyclopedia of Genes and Genomes pathway (KEGG) (18) enrichment analysis to identify functional attributes. GO and KEGG analysis was done using the "DOSE" (19), “org.Hs.eg.db" (20), "clusterProfiler" (21), and "pathview" package (22) in R. For the visualization of the data, the "ggplot2" package (23) was used.

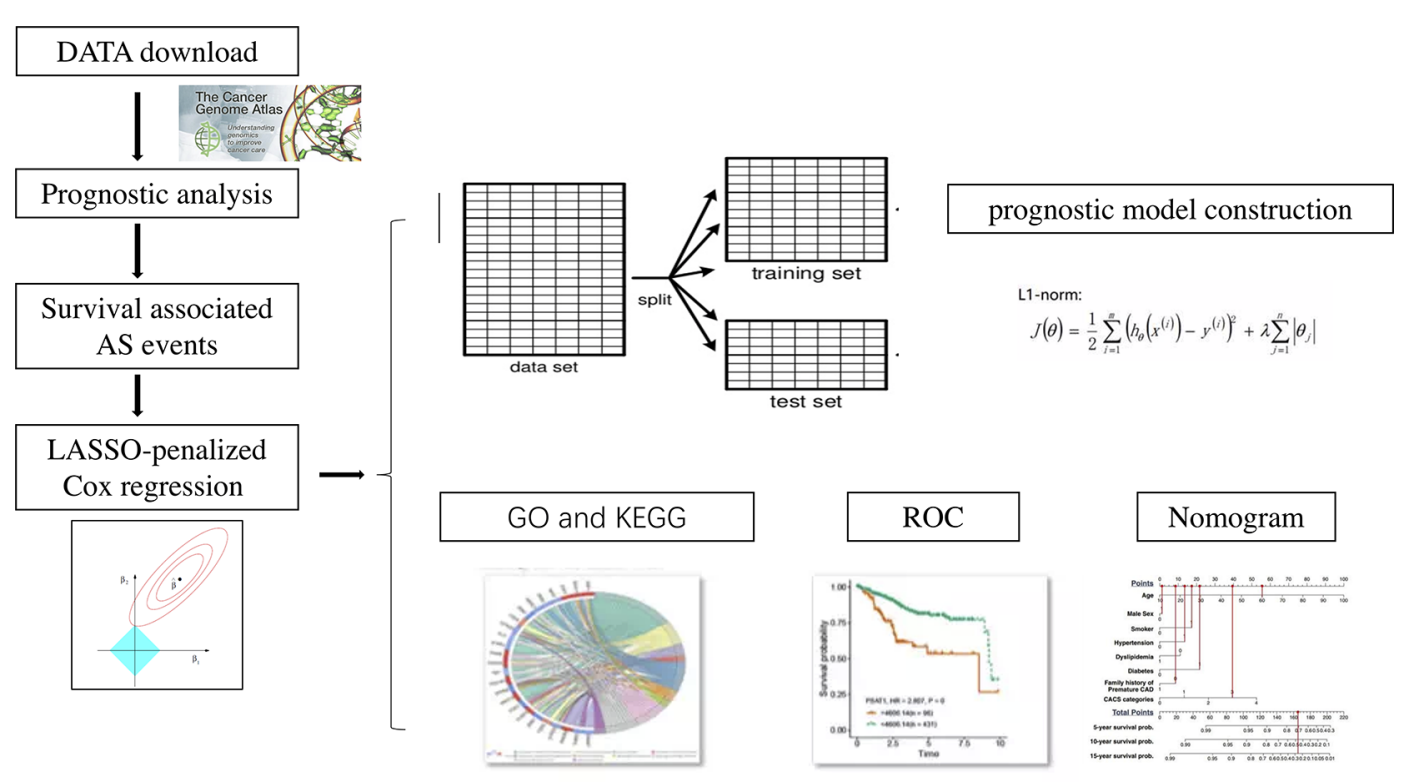

FIGURE 1 | The flow chart for the experimental design. 


\section{Construction of the Nomogram}

Nomogram were generated using both the riskscore value and clinicopathological factors for predicting patient survival probability at 1 and 3 years. Nomogram provide a quantitative and intuitive method to assess the association between variables and survival. Each value within these variables was assigned a score on the point scale. Locate the sum on the Total Points scale and vertically project it onto the bottom axis, we were easily determined the estimated probability of the 1- and 3-year survival probability. Nomograms plots were done with the "rms" package (24) and the "survival" package (15).

\section{RESULTS}

\section{AS Events Profiles in TCGA ACC Cohort}

RNA-seq files and clinical information of 79 ACC patients from TCGA database were downloaded. Two patients without complete follow-up information or complete clinical information were excluded. A total of 34,419 mRNA splicing events in 8,993 genes were detected, which contains 124 MEs in 122 genes, 2,395 RIs in 1,605 genes, 2,707 AAs in 1,960 genes, 2,382 ADs in 1,688 genes, 6,341 APs in 2,575 genes, 8,201 ATs in 3,575 genes, 12,269 ESs in 5,336 genes. These results indicated one gene has several types of splicing events. Among them, ES events were the most common type.

\section{Identify Survival-Associated AS Events}

The association of the alternative splicing events with overall survival was studied by univariate survival analyses. Multiple testing correction was done using Bonferroni adjustment. A total of 1,005 survival-associated alternative splicing events (p.adj < 0.05) were detected. Two ME events in 2 genes, $63 \mathrm{RI}$ events in 58 genes, $54 \mathrm{AA}$ events in 49 genes, $61 \mathrm{AD}$ events in 63 genes, $117 \mathrm{AP}$ events in 74 genes, $325 \mathrm{AT}$ events in 180 genes, and $383 \mathrm{ES}$ events in 330 genes were identified as prognosis-associated AS events (Figure 2A). UpSet plot (Figure 2B) vividly revealed that ES was the predominant event. There were four genes that have three prognosis-related events (ATXN2L, MEIS1, IKBKB, COX4I1).

\section{Molecular Characteristics of Genes With Survival-Associated AS Events}

To reveal the molecular characteristics of genes with survivalassociated AS events, GO and KEGG analyses were conducted (Figure 3). KEGG analysis showed metabolic pathways was the most significant enriched pathway for genes with survivalassociated AS events was Wnt signaling pathway (Figure 3B).

\section{Prognostic Predictors for ACC Patients}

We randomly divided the data set into a training set and an internal validation set according to a 17:3 ratio. First, LASSO analysis was undertaken to identify key prognostic marker to develop prognostic model in alternative splicing seven types (Figure 4A). Twelve alternative splicing events were retained according to the optimal lambda value (Figure 4B, optimal Lambda $\cdot \min =0.2187$ ).

Then, we built the final prognostic predictors including the independent prognostic AS events. The formula for the riskscore

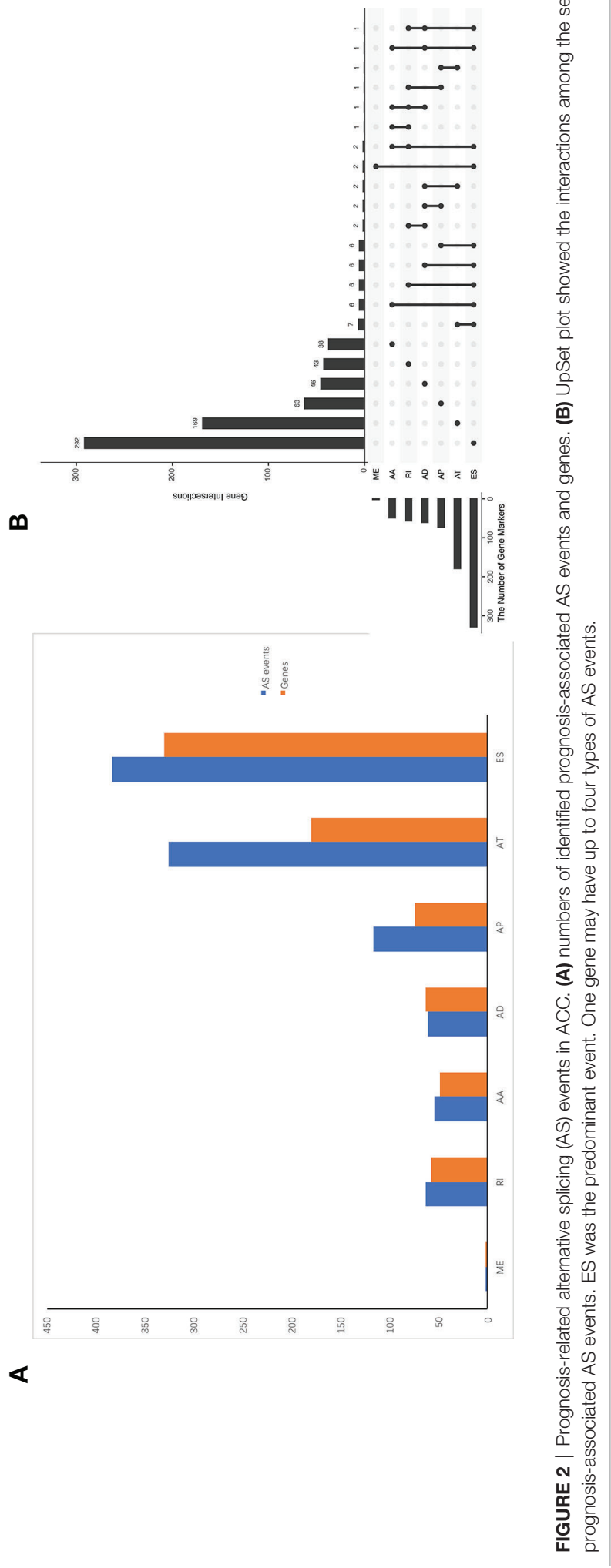




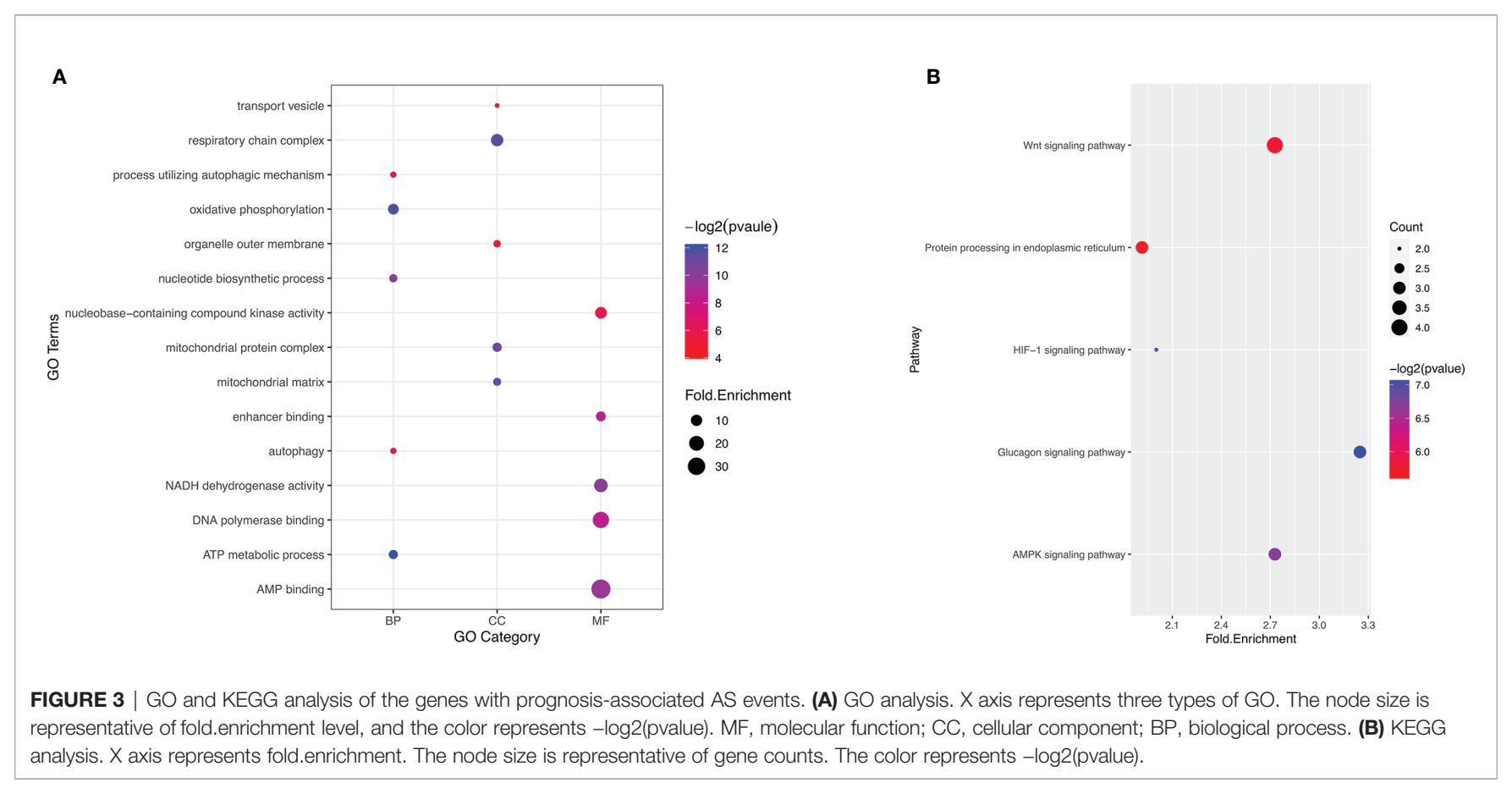

A

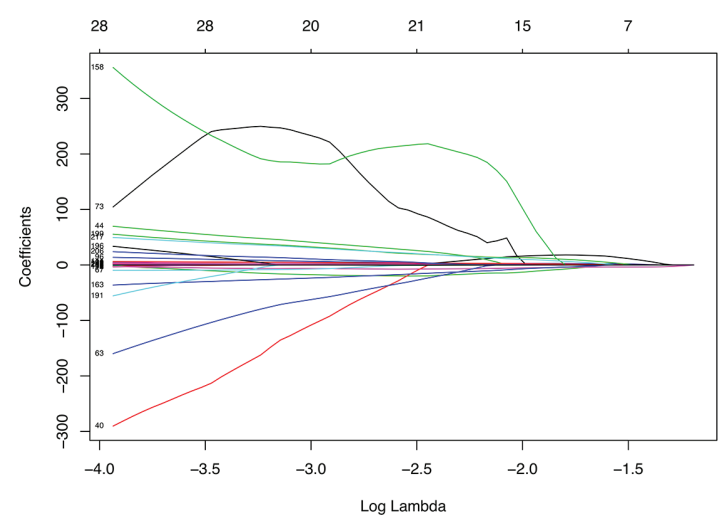

B

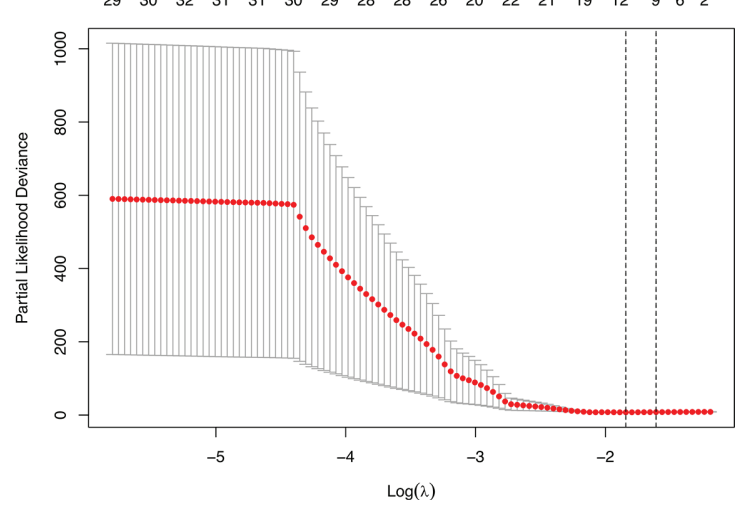

FIGURE 4 | Parameter selection in LASSO analysis. (A) The relationship between the coefficient and the Log(Lambda). The numbers above the graph represent the number of AS involved in the LASSO model. (B) Tuning parameter selection in the LASSO model. The partial likelihood deviance is plotted against Log(Lambda), where Lambda is the tuning parameter. Partial likelihood deviance values are shown, with error bars representing s.e. The dotted vertical lines are drawn at the optimal values by minimum criteria and 1 - s.e. criteria.

can be found in Supplementary Table 1. The riskscore for each patient was showed in Supplementary Tables $\mathbf{2}$ and $\mathbf{3}$. The patients were classified into low-risk group and high-risk group according to the median value of the risk scores. Figure 5 showed patients in high-risk group had poor survival. Then, we applied ROC curves to compare the efficiency of prognostic models. Result showed the prognostic model had a good performance in distinguishing good or poor survival in patients (Figures 6A, B, AUC $=0.92$ and 0.94 in train set and test set, respectively). The KM analysis showed significant difference of survival between high- and low-risk group in both train set and test set (Figures 6C, D).

\section{Construction of a Nomogram for Predicting 1- and 3-Year Survival}

In order to better apply the risk signature, we collected 77 ACC patients with detailed clinical information including $\mathrm{T}$ status, $\mathrm{N}$ status, M status, TNM stage, and gender. Subsequently, a nomogram integrating the five factors and riskscore was constructed for predicting 1- and 3-year survival rate (Figure 7). In the nomogram, the patients' 1 - and 3-year survival rates were estimated by the total points obtained by adding up the point of each factor. 
A

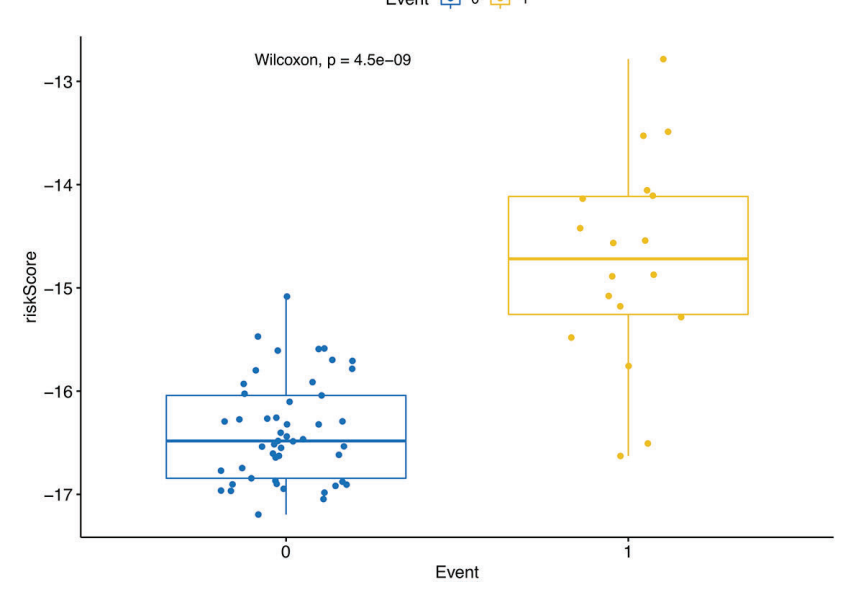

B

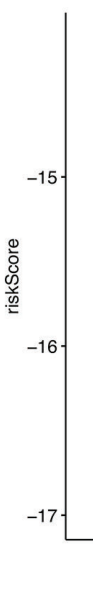

Event 부 0 亩 1

Wilcoxon, $p=0.01$
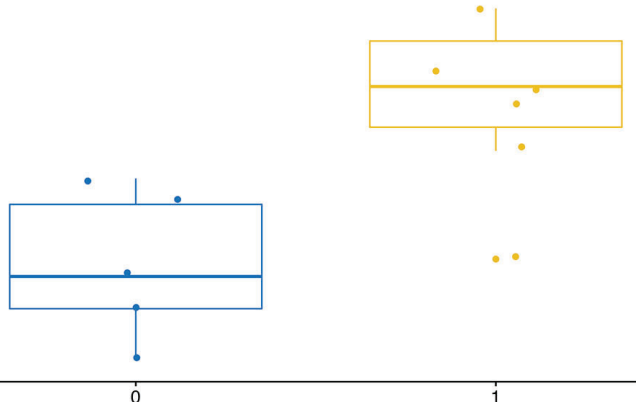

Event

FIGURE 5 | (A) RisksRiskScore of patients with different survival status in train set. 0 representing death and 1 representing alive. (B) RisksRiskScore of patients with different survival status in test set. 0 representing death and 1 representing alive.

A

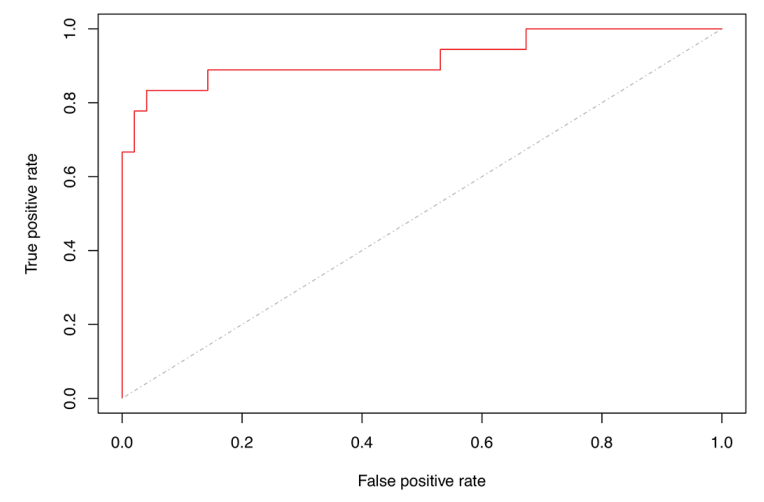

C
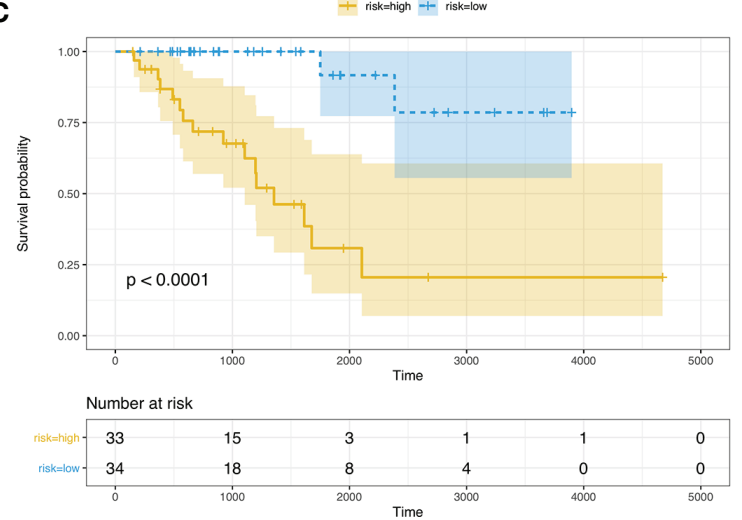

B

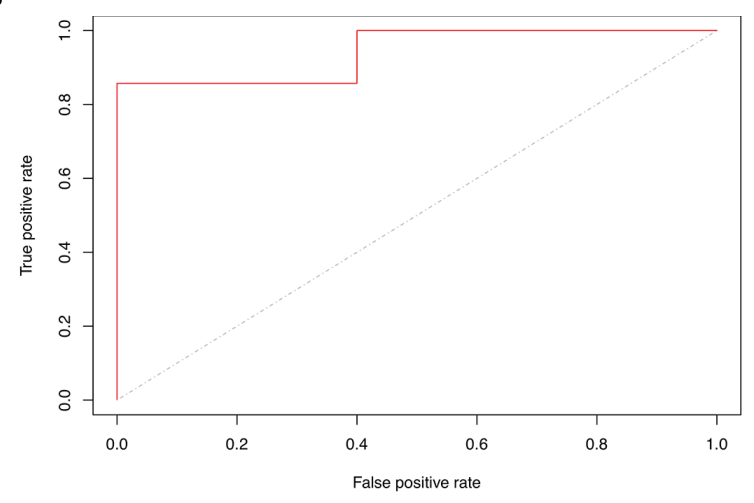

D

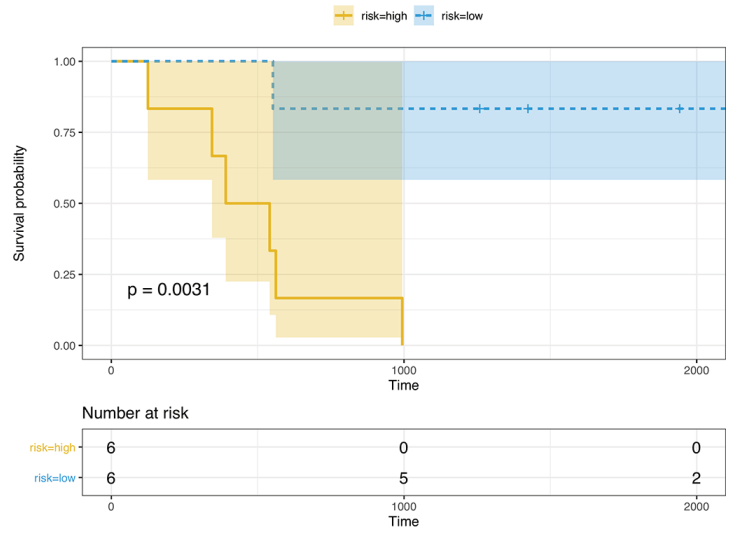

FIGURE 6 | (A) ROC curve of prognostic predictors for ACC in test set. (B) ROC curve of prognostic predictors for ACC in test set. (C) The KM survival analysis of highand low-risk group in train set. Yellow curve, high-risk patient group; blue curve, low-risk patient group. (D) The KM survival analysis of high- and low-risk group in test set. Yellow curve, high-risk patient group; blue curve, low-risk patient group. 


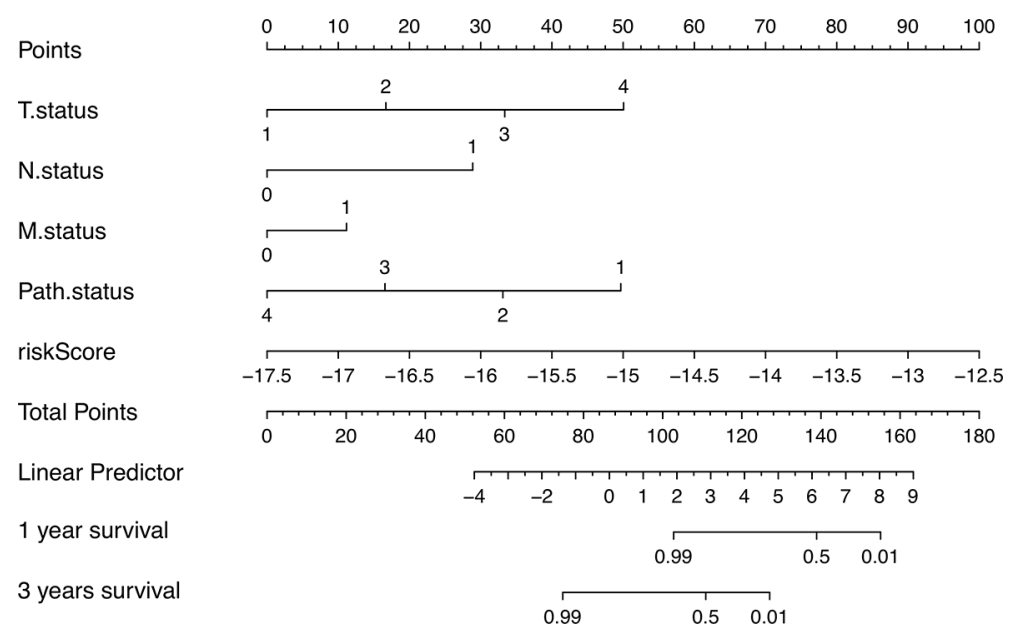

FIGURE 7 | The nomogram for predicting 1- and 3-year survival.

\section{DISCUSSION}

Alternative splicing is an important biological process. Proteincoding genes were limited, it is alternative splicing that provides the potential to generate diversity at RNA and protein levels. Alternative splicing changes are frequently observed in cancer. For example, Goldstein's study showed an alternative splicing change in NFE2L2 leads to the loss of a protein domain and the interaction with its negative regulator $K E A P 1$, thereby providing an alternative mechanism for the activation of an oncogenic pathway. There were studies showing alternative splicing closely associated with cancer patients' survival. Survival-associated alternative splicing had been identified in other types of cancer like lung cancer, thyroid cancer.

To our knowledge, it is the first paper focusing on survivalassociated AS in ACC. In this study, we systematically analyzed the survival-associated AS events in 79 ACC patients from TCGA. Survival-associated AS events were identified. We built well performed prognostic predictors.

In our study, we identified several prognostic genes and potential molecular function. The prognostic genes included ATXN2L, MEIS1, IKBKB, COX4I1. Studies showed these prognostic genes played critical roles in cancer biology. For example, Lin's study showed ATXN2L upregulated by epidermal growth factor promotes gastric cancer cell invasiveness (25). Deregulation of MEIS1 had been reported in cancers like prostate cancer (26), ovarian cancer (27), lung cancer (28). MEIS1 transcriptionally regulated the expression of hypoxic tumor markers, namely Hif- $1 \alpha$ and Hif- $2 \alpha$, which had crucial roles in tumorigenesis (29). Meysam's study showed Msi1 is an important activator for Wnt pathways in esophageal squamous cell carcinoma progression and metastasis (30). COX4I1 is a nuclear gene that encodes the common isoform of cytochrome $\mathrm{c}$ oxidase (COX) subunit 4 ( $\mathrm{COX} 4-1)$, which is an integral regulatory part of the mitochondrial respiratory chain (31). COX4 was a biomarker for breast cancer (32) and medullary thyroid cancer (33). IKKBK, a key molecule in signaling to the transcription factor $\mathrm{NF \kappa B}$ (34), is a very important regulator in tumorigenesis (35-39). Further investigation is necessary to clarify underlying biological links between these genes and ACC. Further investigation is necessary to clarify underlying biological links between these genes and ACC.

Adrenocortical carcinoma (ACC) is an endocrine malignancy with dismal prognosis. Current knowledge of predictors of survival in adrenocortical carcinoma was limited to clinical parameters like increasing age, higher comorbidity index, grade, and stage of ACC (40). Studies of molecular prognostic markers of ACC are limited. Lacombe et al. reported sterol-O-acyl transferase 1 as a prognostic marker (41). A simple prognostic marker based on the differential expression of two genes (BUB1B-PINK1) was developed by de Reyniès (42). Three studies investigated DNA methylation profiles in ACC and showed that a subset of ACC presents hypermethylation of CpG islands (43-45).

Genomics, transcriptomics, proteomics, metabolomics, and other omics technologies have been identified as key technologies to find answers to unresolved clinical questions. There were many studies attempting to dig meaningful information from the large volume data. Predictive model is a good entry point for basic medicine to clinical medicine. One problem of the traditional variable selecting methods such as cox regression and logistic regression is the over-fitting when facing omics data (46). LASSO regression is a good solution of this problem (47). In our study, we constructed prognostic model with 12 predictors using Lasso regression. The AUC of the prognostic predictors was 0.89 . Our prognostic predictors are promising in providing new perspective to understand ACC heterogeneity and to develop new ACC prognostication strategies. 
There was some limitation of this study. Functional studies that further delineate the biologic basis of the impact of dysregulated AS events in ACC are needed. Another large independent validation cohort should be used to validate our result in the future.

In conclusion, our data provide a comprehensive bioinformatics analysis of AS events in ACC. A well performed prognostic predictors was constructed for risk stratification in ACC. Our study provided biomarkers for disease progression and a potentially rich source of novel therapeutic targets. We believe these findings could contribute to clinical cancer management.

\section{DATA AVAILABILITY STATEMENT}

Publicly available datasets were analyzed in this study. The RNA sequencing expression data and clinical information data of 79 ACC patients were obtained from TCGA data portal (https:// tcga-data.nci.nih.gov/tcga/).

\section{REFERENCES}

1. Climente-Gonzalez H, Porta-Pardo E, Godzik A, Eyras E. The Functional Impact of Alternative Splicing in Cancer. Cell Rep (2017) 20:2215-26. doi: 10.1016/j.celrep.2017.08.012

2. Yang X, Coulombe-Huntington J, Kang S, Sheynkman GM, Hao T, Richardson A, et al. Widespread Expansion of Protein Interaction Capabilities by Alternative Splicing. Cell (2016) 164:805-17. doi: 10.1016/ j.cell.2016.01.029

3. Siegfried Z, Karni R. The role of alternative splicing in cancer drug resistance. Curr Opin Genet Dev (2018) 48:16-21. doi: 10.1016/j.gde.2017.10.001

4. Kerkhofs TM, Verhoeven RH, Van der Zwan JM, Dieleman J, Kerstens MN, Links TP, et al. Adrenocortical carcinoma: a population-based study on incidence and survival in the Netherlands since 1993. Eur J Cancer (Oxford Engl 1990) (2013) 49:2579-86. doi: 10.1016/j.ejca.2013.02.034

5. Assie G, Antoni G, Tissier F, Caillou B, Abiven G, Gicquel C, et al. Prognostic parameters of metastatic adrenocortical carcinoma. J Clin Endocrinol Metab (2007) 92:148-54. doi: 10.1210/jc.2006-0706

6. Kebebew E, Reiff E, Duh QY, Clark OH, McMillan A. Extent of disease at presentation and outcome for adrenocortical carcinoma: have we made progress? World J Surg (2006) 30:872-8. doi: 10.1007/s00268-005-0329-x

7. Fassnacht M, Johanssen S, Quinkler M, Bucsky P, Willenberg HS, Beuschlein F, et al. Limited prognostic value of the 2004 International Union Against Cancer staging classification for adrenocortical carcinoma: proposal for a Revised TNM Classification. Cancer (2009) 115:243-50. doi: 10.1002/ cncr. 24030

8. Bilimoria KY, Shen WT, Elaraj D, Bentrem DJ, Winchester DJ, Kebebew E, et al. Adrenocortical carcinoma in the United States: treatment utilization and prognostic factors. Cancer (2008) 113:3130-6. doi: 10.1002/cncr.23886

9. Else T, Williams AR, Sabolch A, Jolly S, Miller BS, Hammer GD. Adjuvant therapies and patient and tumor characteristics associated with survival of adult patients with adrenocortical carcinoma. J Clin Endocrinol Metab (2014) 99:455-61. doi: 10.1210/jc.2013-2856

10. Ayala-Ramirez M, Jasim S, Feng L, Ejaz S, Deniz F, Busaidy N, et al. Adrenocortical carcinoma: clinical outcomes and prognosis of 330 patients at a tertiary care center. Eur J Endocrinol (2013) 169:891-9. doi: 10.1530/EJE13-0519

11. Jouinot A, Bertherat J. MANAGEMENT OF ENDOCRINE DISEASE: Adrenocortical carcinoma: differentiating the good from the poor prognosis tumors. Eur J Endocrinol (2018) 178:R215-30. doi: 10.1530/EJE-18-0027

12. Ryan M, Wong WC, Brown R, Akbani R, Su X, Broom B, et al. TCGASpliceSeq a compendium of alternative mRNA splicing in cancer. Nucleic Acids Res (2016) 44:D1018-22. doi: 10.1093/nar/gkv1288

\section{AUTHOR CONTRIBUTIONS}

WL analyzed and interpreted the data and was the major contributor in writing the manuscript. FS formatted the figures and tables. All authors contributed to the article and approved the submitted version.

\section{FUNDING}

This paper was funded by Zhejiang Provincial Natural Science Foundation of China [grant number LQ20H160021].

\section{SUPPLEMENTARY MATERIAL}

The Supplementary Material for this article can be found online at: https://www.frontiersin.org/articles/10.3389/fendo.2021. 538364/full\#supplementary-material

13. Ryan MC, Cleland J, Kim R, Wong WC, Weinstein JN. SpliceSeq: a resource for analysis and visualization of RNA-Seq data on alternative splicing and its functional impacts. Bioinformatics (2012) 28:2385-7. doi: 10.1093/ bioinformatics/bts 452

14. Conway JR, Lex A, Gehlenborg N. UpSetR: an R package for the visualization of intersecting sets and their properties. Bioinformatics (2017) 33:2938-40. doi: 10.1093/bioinformatics/btx364

15. Lee DK. Survival analysis: part II - applied clinical data analysis. Korean J Anesthesiol (2019) 72(5):441-57. doi: 10.4097/kja.19183

16. Friedman J, Hastie T, Tibshirani R. Regularization Paths for Generalized Linear Models via Coordinate Descent. J Stat Softw (2010) 33:1-22. doi: 10.18637/jss.v033.i01

17. Huang $\mathrm{R}$, Liao $\mathrm{X}, \mathrm{Li} \mathrm{W}$. Identification and validation of potential prognostic gene biomarkers for predicting survival in patients with acute myeloid leukemia. Onco Targets Ther (2017) 10:5243-54. doi: 10.2147/OTT.S147717

18. Kanehisa M, Furumichi M, Tanabe M, Sato Y, Morishima K. KEGG: new perspectives on genomes, pathways, diseases and drugs. Nucleic Acids Res (2017) 45:D353-61. doi: 10.1093/nar/gkw1092

19. Yu G, Wang LG, Yan GR, He QY. DOSE: an R/Bioconductor package for disease ontology semantic and enrichment analysis. Bioinformatics (2015) 31:608-9. doi: 10.1093/bioinformatics/btu684

20. Carlson M. org.Hs.eg.db: Genome wide annotation for Human. (2019). doi: 10.18129/B9.bioc.org.Hs.eg.db

21. Yu G, Wang LG, Han Y, He QY. clusterProfiler: an R package for comparing biological themes among gene clusters. OMICS (2012) 16:284-7. doi: 10.1089/ omi.2011.0118

22. Luo W, Brouwer C. Pathview: an R/Bioconductor package for pathway-based data integration and visualization. Bioinformatics (2013) 29:1830-1. doi: 10.1093/bioinformatics/btt285

23. Wickham H. ggplot2: Elegant Graphics for Data Analysis. New York: SpringerVerlag (2016)

24. Huang C, Liu Z, Xiao L, Xia Y, Huang J, Luo H, et al. Clinical Significance of Serum CA125, CA19-9, CA72-4, and Fibrinogen-to-Lymphocyte Ratio in Gastric Cancer With Peritoneal Dissemination. Front Oncol (2019) 9:1159. doi: 10.3389/fonc.2019.01159

25. Lin L, Li X, Pan C, Lin W, Shao R, Liu Y, et al. ATXN2L upregulated by epidermal growth factor promotes gastric cancer cell invasiveness and oxaliplatin resistance. Cell Death Dis (2019) 10:173. doi: 10.1038/s41419019-1362-2

26. Bhanvadia RR, VanOpstall C, Brechka H, Barashi NS, Gillard M, McAuley EM, et al. MEIS1 and MEIS2 Expression and Prostate Cancer Progression: A Role For HOXB13 Binding Partners in Metastatic Disease. Clin Cancer Res (2018) 24:3668-80. doi: 10.1158/1078-0432.CCR-17-3673 
27. Karapetsas A, Tokamani M, Evangelou C, Sandaltzopoulos R. The homeodomain transcription factor MEIS1 triggers chemokine expression and is involved in CD8+ T-lymphocyte infiltration in early stage ovarian cancer. Mol Carcinog (2018) 57:1251-63. doi: 10.1002/mc.22840

28. Li W, Huang K, Guo H, Cui G. Meis1 regulates proliferation of non-small-cell lung cancer cells. J Thorac Dis (2014) 6(6):850-5. doi: 10.3978/j.issn.2072-1439.2014.06.03

29. Aksoz M, Turan RD, Albayrak E, Kocabas F. Emerging Roles of Meis1 in Cardiac Regeneration, Stem Cells and Cancer. Curr Drug Targets (2018) 19:181-90. doi: 10.2174/1389450118666170724165514

30. Moghbeli M, Rad A, Farshchian M, Taghehchian N, Gholamin M, Abbaszadegan MR. Correlation Between Meis1 and Msi1 in Esophageal Squamous Cell Carcinoma. J Gastrointest Cancer (2016) 47:273-7. doi: 10.1007/s12029-016-9824-6

31. Abu-Libdeh B, Douiev L, Amro S, Shahrour M, Ta-Shma A, Miller C, et al. Mutation in the COX4I1 gene is associated with short stature, poor weight gain and increased chromosomal breaks, simulating Fanconi anemia. Eur $J$ Hum Genet (2017) 25:1142-6. doi: 10.1038/ejhg.2017.112

32. Fuchinoue F, Hirotani Y, Nakanishi Y, Yamaguchi H, Nishimaki H, Noda H, et al. Overexpression of PGClalpha and accumulation of p62 in apocrine carcinoma of the breast. Pathol Int (2015) 65:19-26. doi: 10.1111/pin.12235

33. Bikas A, Jensen K, Patel A, Costello J, Reynolds SM, Mendonca-Torres MC, et al. Cytochrome C Oxidase Subunit 4 (COX4): A Potential Therapeutic Target for the Treatment of Medullary Thyroid Cancer. Cancers (Basel) (2020) 12. doi: $10.3390 /$ cancers 12092548

34. Schmid JA, Birbach A. IkappaB kinase beta (IKKbeta/IKK2/IKBKB)-a key molecule in signaling to the transcription factor NF-kappaB. Cytokine Growth Factor Rev (2008) 19:157-65. doi: 10.1016/j.cytogfr.2008.01.006

35. Mitchell S, Vargas J, Hoffmann A. Signaling via the NFkappaB system. Wiley Interdiscip Rev Syst Biol Med (2016) 8:227-41. doi: 10.1002/wsbm.1331

36. Tang X, Jin L, Cao P, Cao K, Huang C, Luo Y, et al. MicroRNA-16 sensitizes breast cancer cells to paclitaxel through suppression of IKBKB expression. Oncotarget (2016) 7:23668-83. doi: 10.18632/oncotarget.8056

37. Wu H, Wang G, Wang Z, An S, Ye P, Luo S. A negative feedback loop between miR-200b and the nuclear factor-kappaB pathway via IKBKB/IKK-beta in breast cancer cells. FEBS J (2016) 283:2259-71. doi: 10.1111/febs.13543

38. Page A, Bravo A, Suarez-Cabrera C, Alameda JP, Casanova ML, Lorz C, et al. IKKbeta-Mediated Resistance to Skin Cancer Development Is Ink4a/ArfDependent. Mol Cancer Res (2017) 15:1255-64. doi: 10.1158/15417786.MCR-17-0157

39. Huang Y, Chen G, Wang Y, He R, Du J, Jiao X, et al. Inhibition of microRNA16 facilitates the paclitaxel resistance by targeting IKBKB via NF-kappaB signaling pathway in hepatocellular carcinoma. Biochem Biophys Res Commun (2018) 503:1035-41. doi: 10.1016/j.bbrc.2018.06.113

40. Tella SH, Kommalapati A, Yaturu S, Kebebew E. Predictors of Survival in Adrenocortical Carcinoma: An Analysis From the National Cancer Database. J Clin Endocrinol Metab (2018) 103:3566-73. doi: 10.1210/jc.2018-00918

41. Lacombe AMF, Soares IC, Mariani BMP, Nishi MY, Bezerra-Neto JE, Charchar HDS, et al. Sterol O-Acyl Transferase 1 as a Prognostic Marker of Adrenocortical Carcinoma. Cancers (Basel) (2020) 12. doi: 10.3390/ cancers 12010247

42. de Reynies A, Assie G, Rickman DS, Tissier F, Groussin L, Rene-Corail F, et al. Gene expression profiling reveals a new classification of adrenocortical tumors and identifies molecular predictors of malignancy and survival. J Clin Oncol (2009) 27:1108-15. doi: 10.1200/JCO.2008.18.5678

43. Rechache NS, Wang Y, Stevenson HS, Killian JK, Edelman DC, Merino M, et al. DNA methylation profiling identifies global methylation differences and markers of adrenocortical tumors. J Clin Endocrinol Metab (2012) 97:E100413. doi: 10.1210/jc.2011-3298

44. Fonseca AL, Kugelberg J, Starker LF, Scholl U, Choi M, Hellman P, et al. Comprehensive DNA methylation analysis of benign and malignant adrenocortical tumors. Genes Chromosomes Cancer (2012) 51:949-60. doi: $10.1002 /$ gcc. 21978

45. Barreau O, Assie G, Wilmot-Roussel H, Ragazzon B, Baudry C, Perlemoine K, et al. Identification of a $\mathrm{CpG}$ island methylator phenotype in adrenocortical carcinomas. J Clin Endocrinol Metab (2013) 98:E174-84. doi: 10.1210/jc.2012-2993

46. Li Y, Sun N, Lu Z, Sun S, Huang J, Chen Z, et al. Prognostic alternative mRNA splicing signature in non-small cell lung cancer. Cancer Lett (2017) 393:40-51. doi: 10.1016/j.canlet.2017.02.016

47. Zhou ZR, Wang WW, Li Y, Jin KR, Wang XY, Wang ZW, et al. In-depth mining of clinical data: the construction of clinical prediction model with $\mathrm{R}$. Ann Transl Med (2019) 7:796. doi: 10.21037/atm.2019.08.63

Conflict of Interest: The authors declare that the research was conducted in the absence of any commercial or financial relationships that could be construed as a potential conflict of interest.

Copyright $(2) 2021$ Liang and Sun. This is an open-access article distributed under the terms of the Creative Commons Attribution License (CC BY). The use, distribution or reproduction in other forums is permitted, provided the original author(s) and the copyright owner(s) are credited and that the original publication in this journal is cited, in accordance with accepted academic practice. No use, distribution or reproduction is permitted which does not comply with these terms. 